REVISTA EUREKA

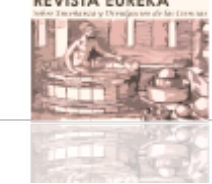

Revista Eureka sobre Enseñanza y Divulgación de las Ciencias

ISSN: 1697-011X

revista.eureka@uca.es

Universidad de Cádiz

España

Propuesta educativa para promover compromisos ambientales a través de los Objetivos de Desarrollo Sostenible en Bachillerato: el juego S.O.S Civilizaciones

González-Robles, Ana; Vázquez-Vílchez, Mercedes

Propuesta educativa para promover compromisos ambientales a través de los Objetivos de Desarrollo Sostenible en Bachillerato: el juego S.O.S Civilizaciones

Revista Eureka sobre Enseñanza y Divulgación de las Ciencias, vol. 19, núm. 1, 2022

Universidad de Cádiz, España

Disponible en: https://www.redalyc.org/articulo.oa?id=92068491015

DOI: https://doi.org/10.25267/Rev_Eureka_ensen_divulg_cienc.2022.v19.i1.1103 


\section{Propuesta educativa para promover compromisos ambientales a través de los Objetivos de Desarrollo Sostenible en Bachillerato: el juego S.O.S Civilizaciones}

Educational proposal to promote environmental engagement through the Sustainable Development Goals in Secondary Education: S.O.S Civilisations game

Ana González-Robles

Departamento de Biología Animal, Biologia Vegetaly

Ecología. Universidad de Jaén, España

agrobles@ujaen.es

(iD) https://orcid.org/0000-0003-3135-8905

Mercedes Vázquez-Vilchez

Departamento de Didáctica de las Ciencias Experimentales.

Universidad de Granada, España

mmvazquez@ugr.es

iD https://orcid.org/0000-0003-4433-5112
DOI: https://doi.org/10.25267/

Rev_Eureka_ensen_divulg_cienc.2022.v19.i1.1103

Redalyc: https://www.redalyc.org/articulo.oa?

$\mathrm{id}=92068491015$

Recepción: 13 Abril 2021

Revisado: 15 Septiembre 2021

Aprobación: 05 Noviembre 2021

\section{Resumen:}

En este trabajo se presenta el diseño y evaluación de un recurso educativo del tipo juego de mesa, titulado 'S.O.S. Civilizaciones'. El diseño de este recurso está basado en el juego comercial "La Isla Prohibida”. Fundamentándose en el aprendizaje basado en juegos, su objetivo es contribuir a la formación y conocimiento del alumnado de Bachillerato en problemáticas medioambientales y sociales del s. XXI, a través de los Objetivos de Desarrollo Sostenible (ONU). La capacidad del juego diseñado para fomentar la motivación y el compromiso pro-ambiental ha sido evaluada mediante un análisis cualitativo utilizando dos marcos teóricos.

Palabras ClaVe: Aprendizaje basado en juegos, compromiso social, educación ambiental, juegos de mesa, Objetivos de Desarrollo Sostenible.

\section{Abstract:}

This paper presents the design and evaluation of a board game type educational resource, entitled 'S.O.S. Civilizations'. The design of this resource is based on the commercial game "The Forbidden Island". Based on game-based learning, its objective is to contribute to the training and knowledge of High School students in environmental and social issues of the 21 st century, through the Sustainable Development Goals (UN). The ability of the designed game to foster motivation and pro-environmental commitment has been evaluated through a qualitative analysis using two theoretical frameworks.

KEYWORDS: Game-based learning, social engagement, environmental education, board gam, global goals.

\section{INTRODUCCIÓN}

En los últimos años, la gamificación ha recibido una gran atención en diversas áreas de investigación (Sailer y Hommer 2020) e incluso está presente en nuestra vida cotidiana, aunque a veces ni siquiera la reconocemos (Dias 2017). Concretamente, la gamificación se ha instalado con fuerza en diferentes áreas como son empresariales (Behl, Sheorey, Pal y Veetil 2020), salud (Sardi, Idri y Fernández-Alemán 2017), medio ambiente (Larson 2020) y, entre otros, el área educativa (Gómez Trigueros 2018). Los numerosos trabajos publicados sobre gamificación proponen diferentes definiciones de este concepto, la más usada en el 
mundo académico es el uso de técnicas y estrategias de juegos en contextos no lúdicos (Deterding, Khaled, Nacke y Dixon 2011).

En el ámbito educativo, la gamificación es una técnica que propone dinámicas asociadas al diseño de juegos en el entorno educativo, con el fin de estimular y tener una interacción directa con los alumnos, permitiéndoles desarrollar sus competencias curriculares, cognitivas y sociales. El uso de la gamificación permite que los estudiantes se involucren y motiven en las tareas y promueve el aprendizaje y la resolución de problemas (Alsawaier 2018). Un caso especial, es el aprendizaje basado en juegos, en el que se utilizan juegos, ya creados o inventados para la ocasión, con el fin de poder aprender a través de ellos, consiguiendo aprendizajes definitivos (Shaffer, Halverson, Squire y Gee 2005).

El aprendizaje basado en juegos es un término que ha ido tomando fuerza en el mundo de la educación en los últimos años (Gómez Trigueros 2018), siendo un campo con una gran cantidad de adeptos y que ofrece un abanico de posibilidades casi infinito para el docente. Dentro del mundo del aprendizaje basado en juegos, existe una rama de especial utilidad para los docentes de las disciplinas de ciencias. Hablamos de los llamados, en inglés, serious games (juegos serios, en su traducción al castellano). Este término se usa para hacer referencia aquellos juegos cuya finalidad no es entretener, sino enseñar, además de crear algún tipo de conciencia, sensibilidad, compromiso o cambio de comportamiento en sus participantes (Schmidt, Radchuk y Meinhart 2014). Buscan hacer de sus jugadores personas implicadas con los problemas actuales, generalmente de índoles medioambiental, económica, social o cívica (ej. Ouariachi, Olvera Lobo y Gutiérrez Pérez 2017; Baaden, Delalande, Ferey, Pasquali, Waldispühl, y Taly 2018). Hablamos así de juegos de participación activa y en los cuales el diálogo y escucha entre los distintos jugadores fundamenta el éxito del juego, al mismo tiempo que el aprendizaje y formación del alumnado. Dado que además este tipo de juegos potencian el pensamiento crítico y sistémico, el razonamiento y la puesta en común de ideas, son elementos idóneos para ser empleados como recursos educativos dentro de las asignaturas de ciencias (ej. Monroe, Oxarart y Plate 2013; Ouariachi et al. 2017; Baaden et al.2018).

Dentro de este marco, los juegos serios fundamentados en el cambio climático han sufrido un drástico auge en los últimos tiempos, debido a la necesidad de hacer llegar a una mayor audiencia los problemas que acarrea y mejorar así la implicación e interés por ellos (Monroe et al. 2013; Wu y Lee 2015). Numerosos países fundamentan gran parte de sus retos gubernamentales en conseguir paliar los efectos antrópicos sobre el medio natural (degradación de hábitat y paisajes, contaminación de aire y agua, introducción de especies invasoras, sobre-explotación de recursos naturales, contaminación de suelos, entre otros), a la par que disminuir las desigualdades sociales. Por ello, si esperan poder conseguir sus objetivos en un futuro próximo resulta indispensable, a la vez que obvio, que aumentar la conciencia, implicación y preocupación de la sociedad por estos temas debe ser una de las primeras metas a conseguir (Wu y Lee 2015). Actualmente, el desarrollo de este tipo de juegos serios dentro de las aulas y centros educativos, es considerada una herramienta muy útil para acercar las problemáticas sociales al público más joven, dotándole de una mentalidad ganadora y optimista, a la vez que comprometida, de tal manera que en su futuro profesional pueda contribuir a conseguir los objetivos ambientales y sociales globales (Corner, Roberts, Chiari, Völler, Mayrhuber, Mandl y Monson 2015; Wu y Lee 2015).

Aunque los juegos que promueven la Educación para la Sosteniblidad han empezado a aplicarse en los entornos educativos, el mundo académico sabe poco sobre cómo funciona el juego con los temas ambientales, cuáles son sus características y su rendimiento real, o cuánto potencial tiene para crear conciencia, compromiso y cambio de comportamiento. Además, pocos marcos teóricos proporcionan una visión integral de los factores que deben considerarse en el diseño de juegos para promover la motivación y el compromiso pro-ambiental (ej. el definido por Chou 2015; o el de Ouariachi, Olvera Lobo y Gutiérrez Pérez 2018). 


\section{OBjetivos}

El presente trabajo presenta un recurso educativo del tipo juego de mesa que pretende contribuir a la formación y conocimiento del alumnado de centros de bachillerato en las problemáticas medioambientales actuales, a través de los Objetivos de Desarrollo Sostenible (en adelante ODS) y la puesta en valor de los mismos. Además, con este juego se pretende que los alumnos se motiven y adquieran un compromiso proambiental ante los diferentes problemas que afectan al Planeta y que nos permitan promover la sostenibilidad en nuestra sociedad. Los retos incluidos en el juego han sido ajustados al nivel y currículum de $2^{\circ}$ Bachillerato de la asignatura Ciencias de la Tierra y del Medio Ambiente (CTMA). Los objetivos educativos concretos que se pretenden conseguir en el alumnado son: i) adquirir conocimientos relacionados con medioambiente, sostenibilidad, bienestar y salud; ii) conocer la existencia y significado de los ODS y iii) comprender la interconexión existente entre todos estos factores.

En este trabajo también se evalúa la capacidad que tiene el juego elaborado para motivar al alumnado y crear compromiso pro-ambiental mediante un análisis cualitativo, utilizando dos marcos teóricos diferentes: marco Octalysis (definido por Chou 2015) y el marco de Compromiso en el Cambio Climático a través de juegos serios (Ouariachi et al. 2018). Concretamente, la evaluación de este recurso persigue conocer la capacidad que tiene este juego de mesa para potenciar la motivación en los usuarios y generar compromisos pro-ambientales profundos en el estudiantado respecto a los diferentes problemas medioambientales que afectan a la sostenibilidad del Planeta, como son, no sólo el cambio climático, sino todos aquellos vinculados con los ODS.

\section{EL JUEGO: S.O.S. CIVILIZACIONES}

El juego propuesto está basado en el juego colaborativo comercial La Isla Prohibida creado por Matt Leacock y distribuido por Devir Iberia y GameWright ${ }^{\circ}$. El juego S.O.S. civilizaciones es un juego colaborativo de 2 a 4 personas, en el que los estudiantes tienen que trabajar en equipo para recuperar cuatro llaves-insignia, para poder huir antes de que el tablero, que es una isla, se hunda. Así los jugadores habrán logrado salvar el mundo y a sus civilizaciones, al haber instaurado en todos los países los ODS. Este recurso está diseñado para que los estudiantes jueguen una partida durante una clase (aproximadamente 1 hora) en grupos de 2 a 4 personas, aunque pueden agruparse en mayor numero. Si bien es recomendable, de acuerdo con Wouters, van Nimwegen, van Oostendorp y van der Spek (2013), que se extienda el tiempo de uso de este recurso y así multiplicar el tiempo de aprendizaje, debido a que uno de los principales objetivos de este juego es promover la comprensión de los complejos problemas ambientales, incluyendo sus causas, impactos y posibles acciones, todo ello a través de los ODS.

El juego consta de varios elementos: dos mazos de cartas (Mazo ODS y Mazo de Inundación), llaves insignias y las losetas de la Isla. Estos elementos están agrupados por 4 familias de diferente color (Figura 1): Agua (azul), Tierra (verde), Sostenibilidad (roja) y Bienestar (naranja). Las cartas ODS presentan retos que deben de ser resueltos por todos los jugadores. Cada familia de cartas ODS permite conseguir una llave insignia determinada (Figura 1). En este mazo de cartas encontramos además cartas especiales (Figura 2): 4 cartas de ¡Suben las aguas!, 4 cartas de Despegamos y 3 cartas de Flotadores. Las cartas ¡Suben las aguas! indican que hay que subir el marcador de las Aguas Suben una unidad, si el nivel sube hasta un determinado nivel, los jugadores pierden. Las cartas de Despegamos permiten desplazarse por la isla y escapar con los 4 tesoros. Las cartas Flotadores permiten reflotar losetas que han sido hundidas, independientemente de su posición en la isla. Por otro lado, el mazo de Inundación presenta cartas que indican qué 'losetas Isla' deben ir hundiéndose a lo largo del juego. Las cartas del mazo de inundación muestran los mismos escenarios que las losetas del juego y están relacionadas con las cartas ODS por colores. (Figura 1). Por último, el juego presenta 6 cartas de Aventurero que definen el rol que toma cada jugador en el juego (Figura 3). 


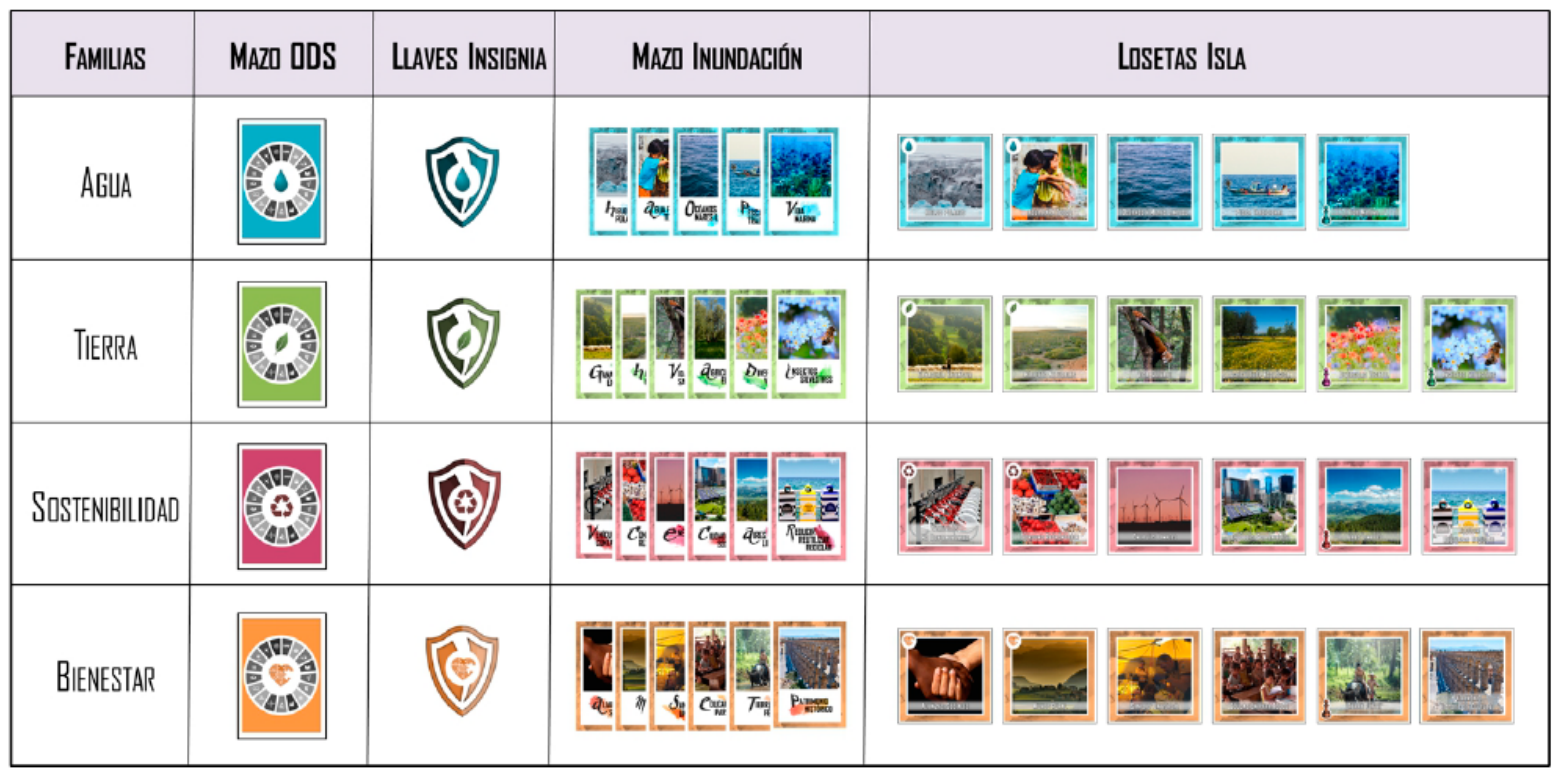

FIGURA 1

Relación por el color entre los diferentes elementos del recurso elaborado y las cuatro familias de ODS diseñadas para el mismo.
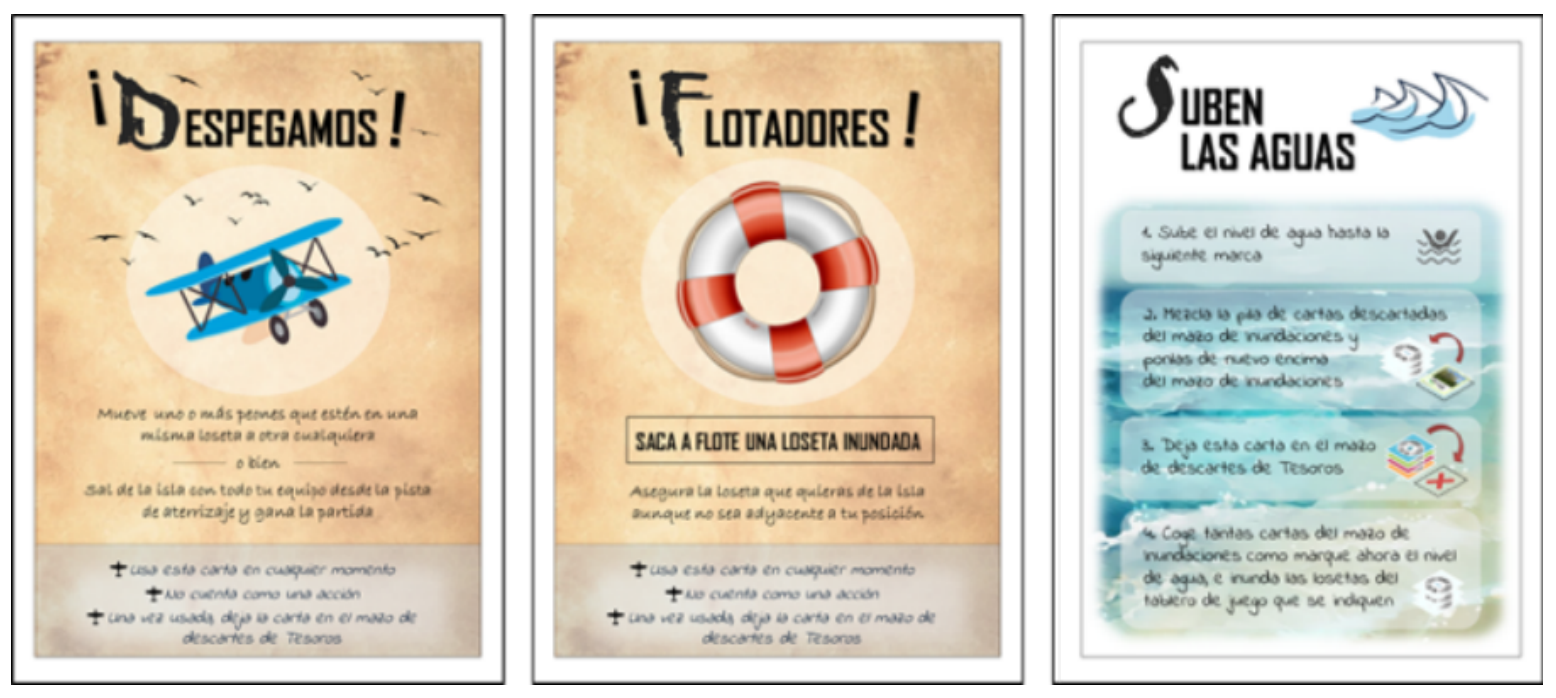

FIGURA 2

Cartas especiales, diseñadas para el recurso educativo, que se encuentran dentro del mazo ODS: i) Despegamos; ii) Flotadores; y iii) Suben las aguas. 


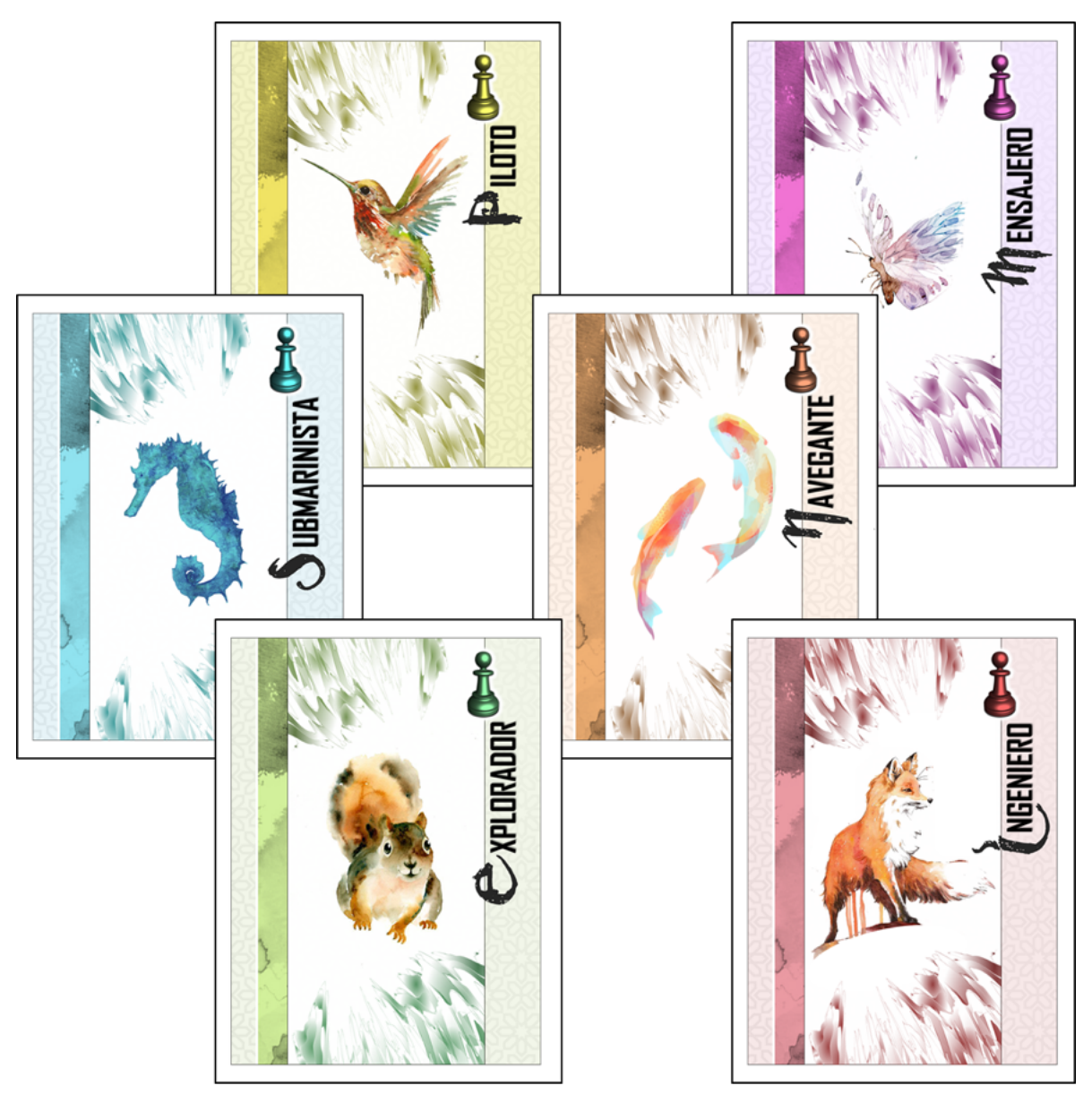

FIGURA 3

Diseño de cada una de las cartas Aventurero del juego.

El tablero está formado por 24 losetas (Figuras 4 y 5). Cada loseta cuenta con dos caras. La cara con tonos de colores se muestra cuando la loseta está a flote, y la cara con tonos ocres y apagados se muestra cuando la loseta está hundida. Las losetas a color muestran los distintos escenarios, prácticas o situaciones con las que se espera que, como ciudadanos, estemos comprometidos socialmente, es decir, muestra un mundo que se implica con los ODS (Figura 4). El lado de color ocre de cada loseta muestra el escenario contrario, es decir, en qué se convierte el mundo si nuestra implicación en los ODS es nula (Figura 5). Las losetas están clasificadas en función de su relación con familias de las cartas de ODS (Figura 1):

- Familia Agua (azul): Océanos y mares limpios (vs. océanos y mares de plástico); agua para todos (vs. sin agua potable); hielos polares (vs. disminución de hielos polares); vida marina (vs. destrucción de la vida marina); pesca tradicional (vs. pesca intensiva).

- Familia Tierra (verde): Agricultura ecológica (vs. agricultura intensiva); ganadería extensiva (vs. ganadería intensiva); diversidad vegetal (vs. no plantas silvestres); insectos silvestres (vs. no insectos); hábitats naturales (vs. destrucción de hábitats); vida salvaje (vs. vida cautiva).

- Familia Sostenibilidad (roja): Aires limpios (vs. aires contaminados); ciudades sostenibles (vs. ciudades no sostenibles); vehículos no contaminantes (vs vehículos contaminantes); energías limpias (vs. energías contaminantes); consumo responsable (vs. consumo excesivo); reducir, reutilizar y reciclar (vs. usar y tirar).

- Familia Bienestar (naranja): Tierra fértil (vs. tierra estéril); alianzas sociales (vs. no alianzas sociales); educación para todos (vs. trabajo infantil); patrimonio histórico y cultural (vs. destrucción de patrimonio); mundo rural (vs. vida urbana); sanidad universal (vs. sin acceso a sanidad). 


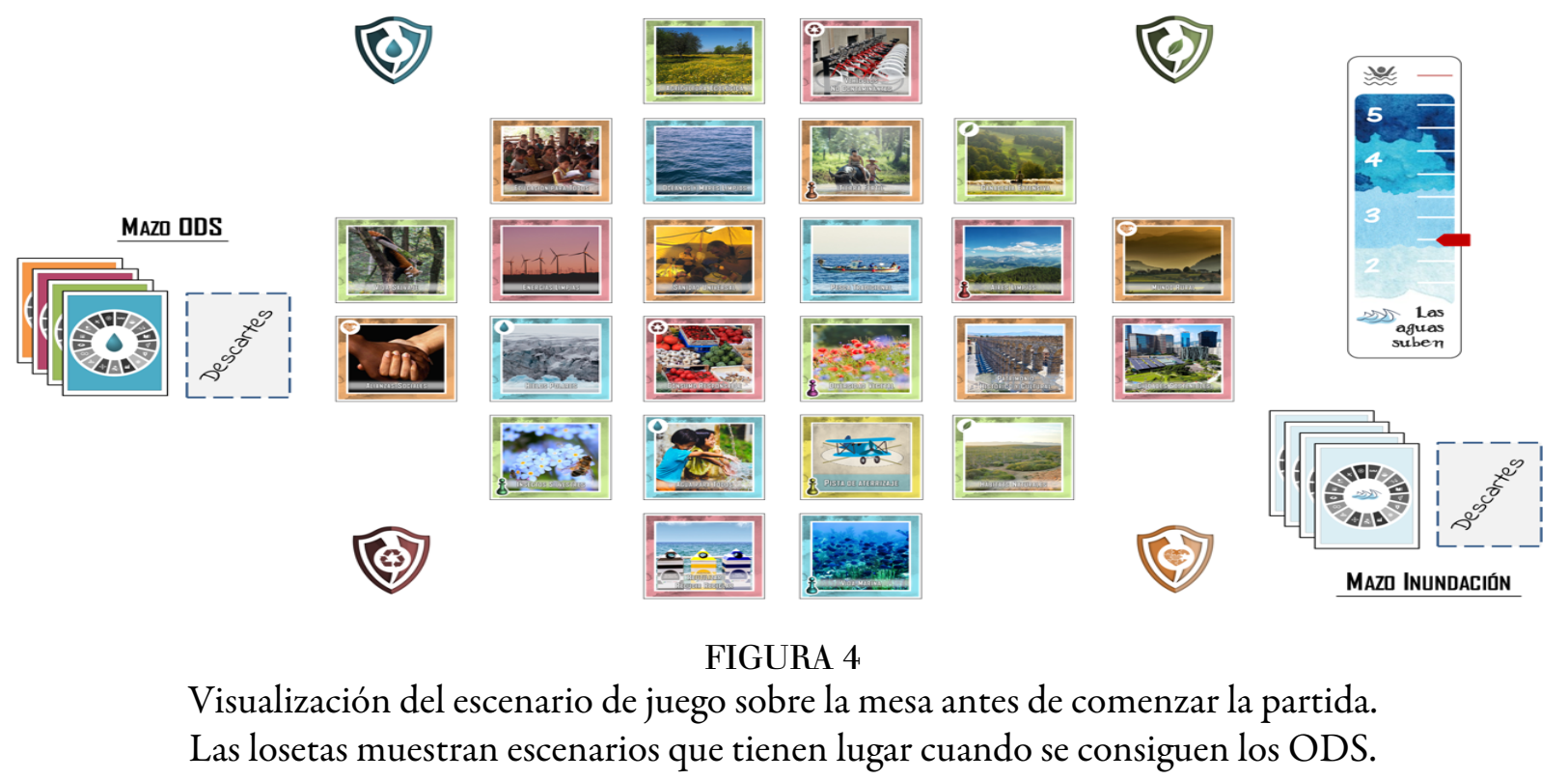

El juego consiste en conseguir las 4 llaves-insignias resolviendo en grupo los retos presentados por las cartas ODS. Cuando un jugador tenga en su poder tres cartas ODS del mismo color podrá recuperar la llave-insignia asociada a dicha familia si está situado en una loseta que lo permita, marcado con el mismo signo insignia (Figura 6). Si los jugadores no resuelven adecuadamente el reto de la carta ODS, subirán el nivel del marcador de las Aguas Suben.

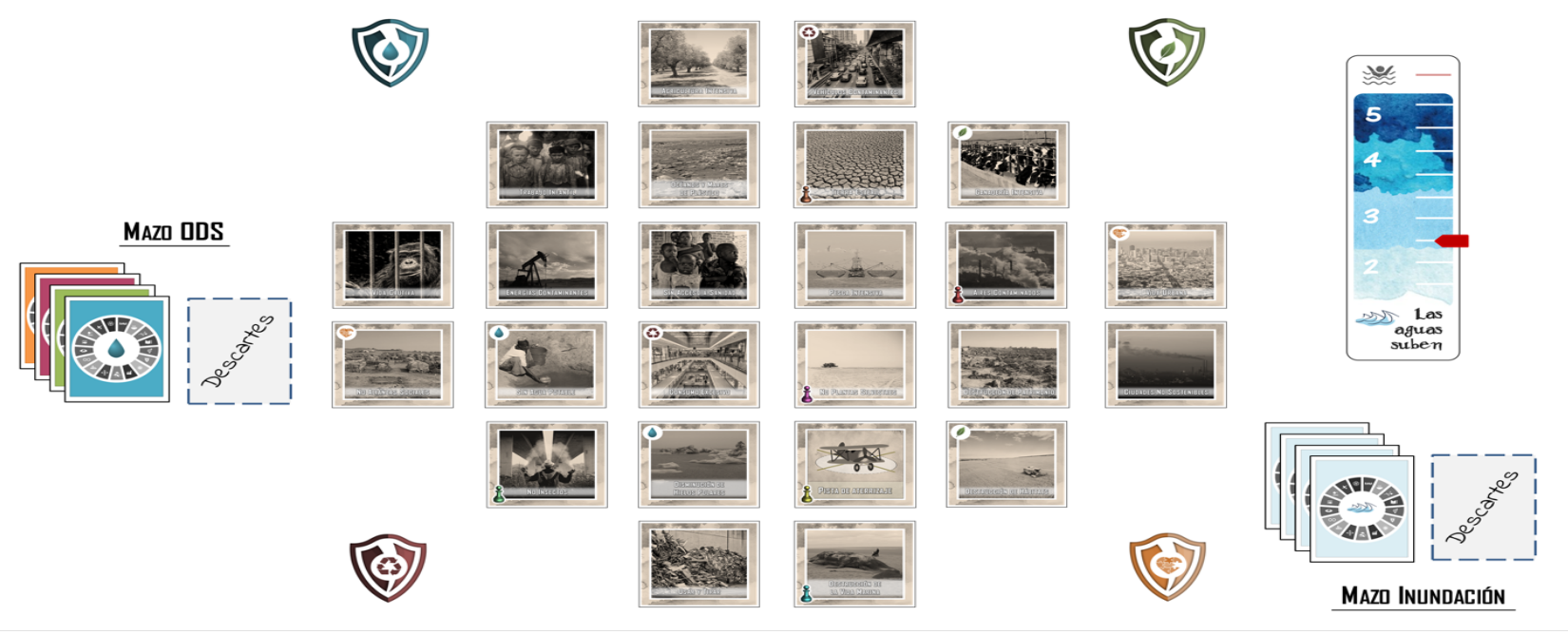

\section{FIGURA 5}

Visualización del escenario de juego sobre la mesa en el caso de que todas las losetas Isla estuvieran hundidas. Muestra escenarios que tienen lugar cuando no se consiguen los ODS.

Los retos a resolver en cada una de las familias ODS, han sido definidos a partir de los Objetivos de Desarrollo Sostenible de Naciones Unidas (Figura 7). Un total de 48 retos han sido diseñados para este juego de mesa. Algunos ejemplos de estos retos se muestran en el Anexo 1. A partir de los ODS, y teniendo en cuenta los contenidos y criterios de evaluación objeto de la asignatura de CTMA de $2^{\circ}$ de Bachillerato (ver Anexo 1), se hizo una subdivisión en grupos de los mismos:

- Valoración y preocupación por el medio ambiente y su biodiversidad: Objetivos 6 (Agua limpia y saneamiento), 13 (Acción por el clima), 14 (Vida submarina), y 15 (Vida de ecosistemas terrestres). Este 
grupo dio lugar a dos familias ODS: Agua (trabajando los objetivos 6 y 14) y Tierra (trabajando los objetivos 13 y 15$)$.

- Impacto de las actividades humanas y sostenibilidad: Objetivos 7 (Energía asequible y no contaminante), 11 (Ciudades y comunidades sostenibles), y 12 (Producción y consumo responsable). Son trabajados dentro de la familia ODS de Sostenibilidad.

- Salud: Objetivo 3 (Salud y bienestar). Se trabaja dentro de la familia ODS de Bienestar.

- Desarrollo e implicación social: Objetivos 1 (Fin de la pobreza), 2 (Hambre cero), 4 (Educación de Calidad), 5 (Igualdad de género), 8 (Trabajo decente y crecimiento económico), 9 (Industria, innovación e infraestructuras), 10 (Reducción de las desigualdades), 16 (Paz, justicia e instituciones sólidas), 17 (Alianzas para lograr los objetivos). Estos objetivos, que guardan mayor relación con temas de implicación social y empáticos, no fueron trabajados como tal dentro del mazo de familias ODS, aunque por su naturaleza son temas que están profundamente relacionados con los objetivos ODS que se trabajan mediante preguntas dentro de las cuatro familias de cartas ODS. Además, la problemática social que motiva la misión de los jugadores, y los escenarios que muestran las losetas Isla, buscan trabajar todos estos temas, creando conciencia en el alumnado, no mediante la búsqueda de información, sino mediante la formación de su pensamiento crítico y empático hacia la sociedad y el mundo. Así, el alumnado trabajará estos objetivos de una forma indirecta, pero muy activa, dado que todos ellos son el fundamento de su misión como jugadores.
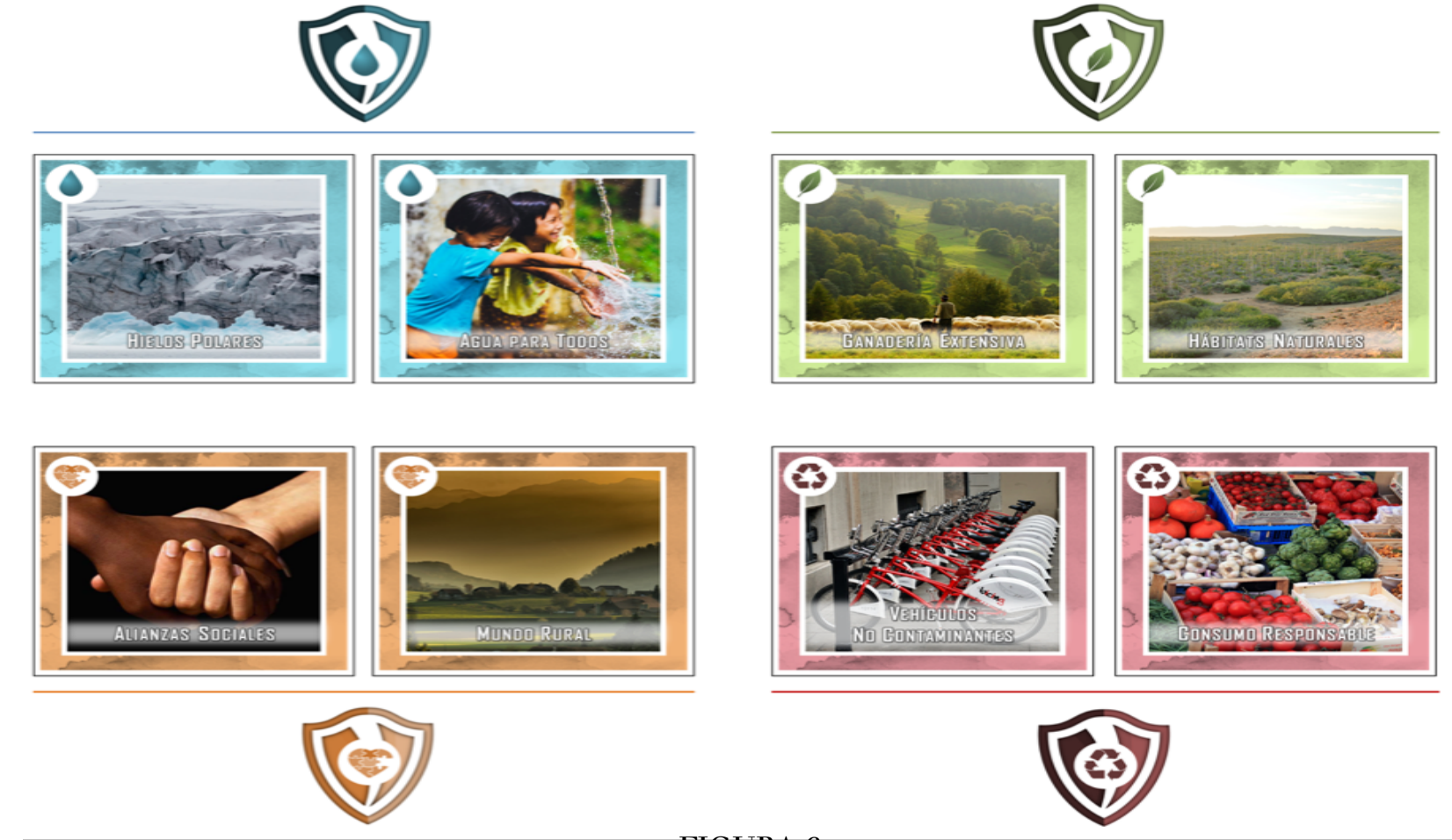

FIGURA 6

Llaves-insignia (Tesoros) asociadas a su pareja de sus losetas en la isla.

Para el diseño de los retos se utilizaron distintas estructuras, con la finalidad de hacer el juego más atractivo a los participantes. De esta forma, encontramos preguntas de tipo: i) verdadero y falso; ii) abiertas de desarrollo y reflexión; iii) completar espacios; iv) buscar relaciones a modo 'une con flechas'; v) interpretación de gráficos e imágenes; vi) acertijos o crucigramas; vii) elección múltiple; y viii) pequeños cálculos (ver Anexo 1). Con la intención de contextualizar las preguntas en ambientes cercanos, muchas de ellas están basadas en experiencias o impresiones propias del alumnado, o en casos dentro de España. Atendiendo a la dinámica del juego elaborado, y al tratarse de un recurso de tipo 'serious game' fundamentado en los juegos de mesa colaborativos, los modelos de pensamiento principalmente trabajados con dicho recurso serían: el reflexivo, el analítico, el lógico, y el crítico. 
Para guiar el proceso de enseñanza y aprendizaje del alumnado durante el transcurso del juego, se han incluido códigos QR en la mayor parte de las cartas de preguntas. Estos códigos dan acceso a videos cortos, divulgativos, de la plataforma web YouTube, o a artículos virtuales que cuentan con información suficiente para resolver las cuestiones que se plantean. A su vez, el profesorado encargado de la asignatura deberá hacer de 'guía de juego' y marcar la dinámica y tiempos del mismo, según las características del alumnado con el que se trabaje.
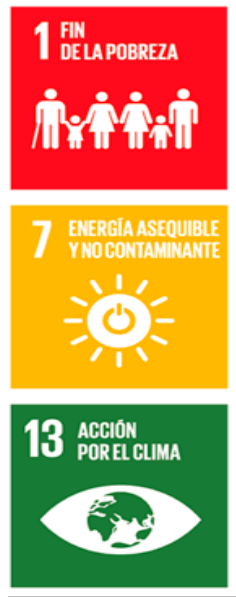
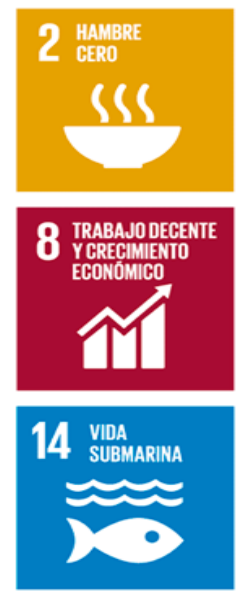
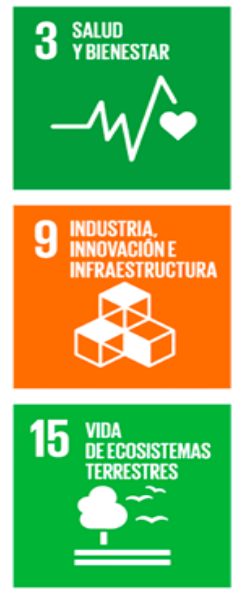
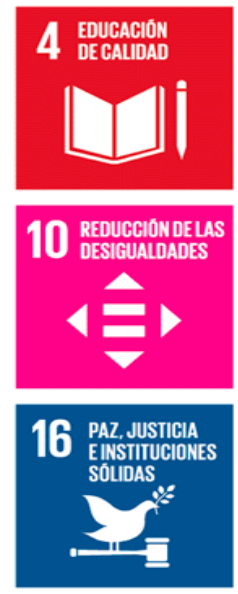
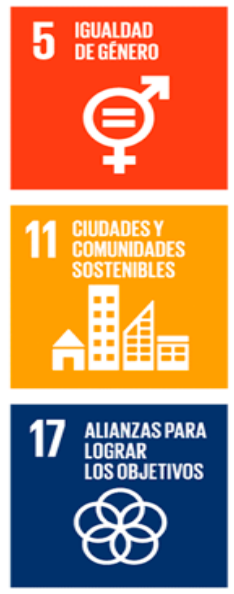
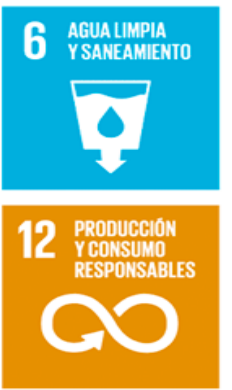

OBJETIVOS

DOSTENIBLE

\section{FIGURA 7}

Los 17 Objetivos de Desarrollo Sostenible para 2030 definidos por Naciones Unidas United Nations website

Cabe mencionar que para la elaboración de las cartas y preguntas ODS, así como para la selección de la información contenida en los códigos QR, se ha hecho especial hincapié en que la información sea visualmente atractiva para el alumnado, con la intención de captar su atención lo máximo posible. Se pretende así que, la utilización de este recurso en las aulas, sea entendida por ellos verdaderamente como un juego, y no como otra clase teórica.

Por último, para la evaluación del conocimiento en medioambiente y sostenibilidad adquirido por el estudiantado, se propone que los estudiantes tras jugar una partida, entreguen de forma individual un Informe de Aventurero, imaginando que deben de entregarlo a la ONU al regresar de su aventura en la Isla, para informales de sus descubrimientos en ella. En este informe los estudiantes deben de recopilar los resultados de los retos a los que se hayan enfrentado durante el juego. De esta manera, el profesorado puede evaluar cada estándar de aprendizaje implicado en los retos del juego. Además también se recomienda evaluar mediante la observación directa la participación y el trabajo en equipo del estudiantado.

\section{Evaluación del Juego S.O.S Civilizaciones}

En este trabajo hemos utilizado un análisis cualitativo de contenidos (Chou y Lee 2014) para evaluar la capacidad de motivación y generación de comportamientos pro-ambientales en relación a los ODS del juego "S.O.S Civilizaciones" en estudiantes de Bachillerato. Dos marcos teóricos diferentes se han utilizado: el marco teórico de Octalysis (Chou 2015) y el marco de compromiso en el Cambio Climático a través de juegos serios (Ouariachi et al. 2018). La integración de estos dos modelos diferentes en el análisis de contenido cualitativo se basa en la necesidad de realizar una triangulación teórica en los estudios exploratorios, pero también en la necesidad de integrar los puntos fuertes de cada modelo. Aunque ambos modelos persiguen el mismo objetivo, el primero se basa sobre todo en la gamificación como herramienta, mientras que el segundo se centra en cuestiones relacionadas con el cambio climático. Cabe destacar, que el juego "S.O.S. Civilizaciones" incluye más aspectos medioambientales que el cambio climático, como son todos 
aquellos relacionados con la perdida/destrucción de la biodiversidad, impacto de las actividades humanas y sostenibilidad.

\section{Marcos teóricos}

\section{Marco teórico de gamificación Octalysis}

El marco teórico Octalysis fue creado por You-Kai Chou en 2015 (Octalysis framework, https://yukaichou.c om) y se define como un marco de gamificación centrado en el ser humano que pretende evaluar la motivación de los usuarios mientras juegan. El marco está dividido en ocho unidades centrales que se centran en aspectos motivacionales separados, pero interrelacionados y conectados al comportamiento humano: i) significado épico y llamada; ii) progresión y desarrollo; iii) creatividad y feedback; iv) posesión; v) influencia social y sentimiento de pertenencia; vi) escasez e impaciencia; vii) curiosidad y azar; y viii) miedo a la perdida.

El significado épico y la llamada se refiere a hacer sentir a los usuarios que sus acciones están conectadas a una causa mayor que ellos mismos. Progresión y desarrollo evalúa la motivación de conocer los progresos logrados, esto lo podemos lograr con la utilización de niveles, barras de progreso, etc. Creatividad y feedback es el núcleo más importante, y consiste en medir la motivación que experimentamos cuando expresamos nuestra creatividad y recibimos un feedback y reconocimiento. La posesión se refiere a la necesidad humana de adquirir posesiones y riquezas. La influencia social y el sentimiento de pertenencia evalúa la capacidad que tiene el juego de permitir relacionarnos y hacernos sentir pertenecientes a una comunidad. Escasez e impaciencia cuantifica la capacidad que tiene el juego de hacernos sentir que conseguimos cosas que no tenemos o que son especiales. Curiosidad y azar mide la capacidad que tiene el recurso de incentivarnos a realizar acciones que nos sorprenden y de las cuales no conocemos el resultado. Finalmente, el miedo a la perdida evalúa el sentimiento de sufrir consecuencias negativas.

Chou clasifica estas 8 unidades de dos formas diferentes. Una clasificación es teniendo en cuenta aquellas que motivan al jugador de forma positiva y otras negativas, las cuales son llamadas unidades de sombrero blanco y de sombreo negro, respectivamente. Las de sombrero blanco son el significado épico y la llamada, progresión y desarrollo, creatividad y feedbak, la influencia social y el sentimiento de pertenencia. Estas unidades o atributos nos hacen sentir bien y poderosos porque nos permiten expresar la creatividad, sentirnos exitosos a través del dominio de nuestras habilidades. Por otro lado, las de sombrero negro son escasez e impaciencia, curiosidad y azar, y finalmente, sentimiento a la perdida. Estas unidades nos hacen sentir ansiosos porque no sabemos lo que va a pasar después, tenemos constantemente miedo de perder algo, o porque hay cosas que no podemos tener, aunque sigamos estando extremadamente motivados para realizar las acciones, a menudo nos pueden dejar un mal sabor de boca. Este autor también clasifica estas unidades como aquellas de motivación extrínsecas o intrínsecas. Las unidades de motivación extrínsecas (progresión y desarrollo, posesión y escasez e impaciencia) son las responsables de que nos sintamos motivados porque quieres obtener algo, ya sea un objetivo, un bien o cualquier cosa que no puedamos obtener. Las unidades de motivación intrínseca (creatividad y retroalimentación, influencia social y sentimiento de pertenencia e imprevisibilidad y curiosidad) están relacionadas con sentimientos agradables producidos por una actividad en sí misma, no necesitamos un objetivo o una recompensa para utilizar nuestra creatividad, o simplemente sentir el suspense de la imprevisibilidad es gratificante por sí mismo.

\section{Marco Teórico de Compromiso en el Cambio Climático a través de Juegos Serios}

Ouariachi et al. (2018) determinaron qué atributos deben tener los juegos para crear compromiso en los usuarios frente al cambio climático. Nosotras hemos aplicado este marco teórico no sólo para evaluar el 
compromiso creado en los jugadores frente al cambio climático sino también frente a otros problemas ambientales que recogen los ODS. De acuerdo con estos autores el compromiso pro-ambiental se puede alcanzar cuando los jugadores logran comprometerse de forma cognitiva, emocional y de comportamiento. Los atributos propuestos por Ouariachi et al. (2018) son los siguientes: 1) retos alcanzables, 2) desafiante; 3) concreto; 4) creíble; 5) que aumenten la eficacia; 6) aprendizaje experimental; 7) orientados a la retroalimentación; 8) divertidos; 9) impulsados por la identidad; 10) de nivelación; 11) significativos; 12) impulsados por la narrativa; 13) impulsados por la recompensa; 14) de simulación; y 15) sociales. Según este marco teórico cuando el diseño de un juego presenta los atributos mencionados presentará un alto potencial para comprometer a la gente de forma cognitiva, emocional y conductual en los problemas ambientales que afectan a nuestro Planeta.

Algunos de estos atributos pertenecen a las tres dimensiones de compromiso, lo que significa que son ejes centrales para potenciar el compromiso cognitivo, emocional y de comportamiento simultáneamente. Estos atributos son: a) objetivos alcanzables: son aquellos objetivos que pueden promover acciones que pueden realizar los estudiantes; b) orientado a la retroalimentación: las decisiones y acciones tomadas en el juego deben de tener una retroalimentación y son deseables de ser replicadas por los estudiantes en la vida real; c) significativo: el juego debe promover emociones intensas; d) conducido por la narrativa: la narrativa del juego debe conectar con los estudiantes, creando una fantasía que sea significativa para el jugador; e) simulador: el juego debe conectar y asociar acciones específicas con consecuencias específicas, ya que pueden resonar en espacios mucho más amplios y en momentos más distantes de lo que el estudiante puede percibir en la vida real.

\section{Metodología}

El marco teórico Octalysis ha sido aplicado a nuestro juego mediante una herramienta en línea (Octalysis tool, https://www.yukaichou.com/octalysis-tool/) en la que las dos investigadoras puntuaban las diferentes unidades (Figura 8). Cada unidad de motivación fue valorada de 0 a 10 puntos, que posteriormente se elevaron al cuadrado y sumaron dando así de forma conjunta la puntuación final del juego. Finalmente, la herramienta proporcionó comentarios del potencial del diseño del juego para motivar a los estudiantes en temas de sostenibilidad.

Por otro lado, el análisis de evaluación utilizando el marco teórico de compromiso en el Cambio Climático a través de juegos serios (Ouariachi et al. 2018) permitió explorar el potencial de nuestro juego en crear compromisos pro-ambientales. En este caso las investigadoras utilizaron una lista de verificación (Figura 9 y Tabla 1) para comprobar la presencia o no de los atributos propuestos en este marco teórico. Para evitar las implicaciones interpretativas, las investigadoras siguieron un protocolo de directrices establecido previamente. Este protocolo de directrices consistió en definir que características referidas a cada atributo están presentes en nuestro juego. Así, se identificó si el juego S.O.S: Civilizaciones fomentaba: a) el desarrollo de retos alcanzables, tales como, la comprensión de que la colaboración permite alcanzar la sostenibilidad; b) el desarrollo de retos concretos, creíbles y desafiantes, como por ejemplo, retos concretos basados en la experiencia de los estudiantes y que requieren un esfuerzo; c) el sentimiento de empoderamiento, haciendo sentir a los estudiantes como héroes que salvan el planeta; d) el aprendizaje experiencial a través de la narrativa e imágenes, permitiendo observar las consecuencias de un mundo no sostenible, por ejemplo en las imágenes de las losetas; e) que las acciones y decisiones tomadas en el juego permitan una retroalimentación que los estudiantes puedan replicar en la vida real, por ejemplo, estimulando la colaboración ciudadana; f) la diversión y el entretenimiento; g) el desarrollo de identidades que los estudiantes desearían ser en el futuro, tales como héroes que salvan el Planeta; h) el desarrollo de sentimientos significativos, positivos, al sentirse héroes, y negativos por el impacto generado con las imágenes que muestran el Planeta como un mundo no sostenible; i) la creación de una fantasía significativa para los jugadores a través de la narrativa del juego, como 
por ejemplo, ser los salvadores del mundo; j) la obtención de la recompensa, que en este caso es salvar el mundo; k) el desarrollo social, fomentando la interacción y actuación por pares, permitiendo intercambiar opiniones, recibir elogios, etc.

\section{Resultados}

\section{Aplicación del Marco Octalysis}

Para obtener la puntuación de Octalysis, se estima la calidad de la unidad sujeta a análisis y se asigna un número entre 0 y 10 basado en el juicio personal y luego se eleva al cuadrado ese número para obtener la puntuación de la unidad. Una vez que se suman las 8 puntuaciones de la unidad central, se obtiene la puntuación final del Octalysis.

La figura 8 muestra los resultados obtenidos tras aplicar el marco de Octalysis. La puntuación obtenida por las dos autoras para el juego elaborado es bastante alta, con valores de 605 y 620 puntos. Las unidades motivacionales de sentido épico y llamada e influencia social y sentimiento de pertenencia son las más fuertes, mientras que las unidades de progresión y desarrollo y posesión son las menos desarrolladas. En cambio, en términos generales, el juego S.O.S Civilizaciones presenta un gran equilibrio entre la motivación intrínseca y la extrínseca.

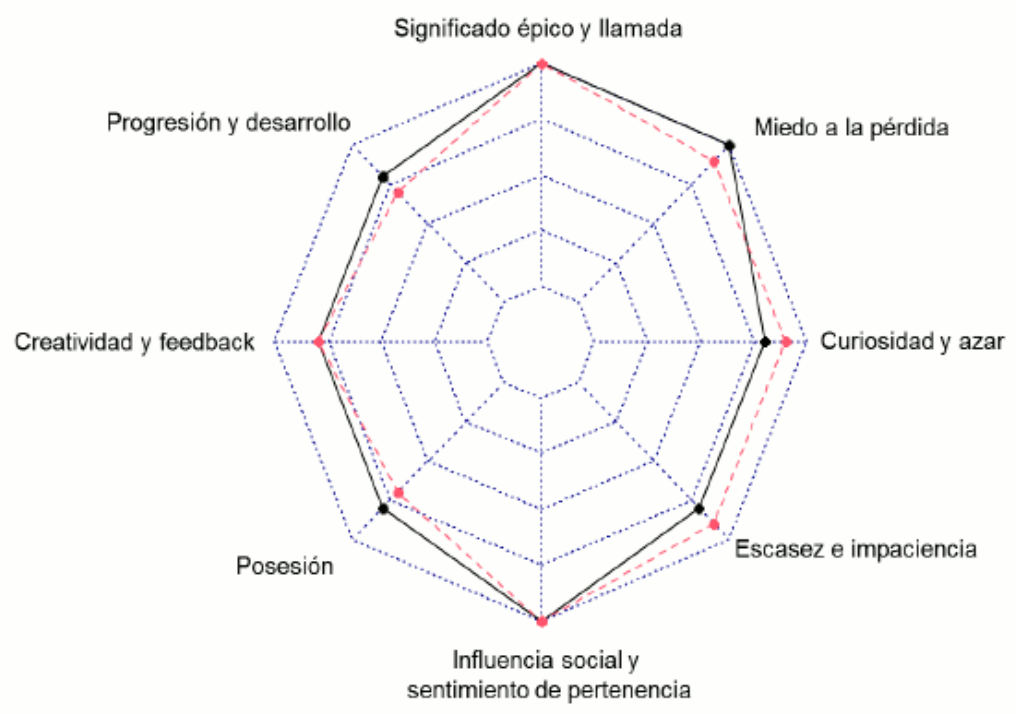

FIGURA 8

Resultados obtenidos según el marco de Octalysis. Los colores rojo y negro muestran la valoración de cada una de las investigadoras. El color azulindica la escala de puntuación en unidades de 2 de cada unidad de valoración sujeta al análisis.

\section{Aplicación del Marco de Compromiso en Cambio Climático a través de Juegos Serios}

El juego propuesto ha sido evaluado teniendo en cuenta el marco de compromiso en el cambio climático a través de juegos serios (Ouariachi et al. 2018) (Figura 9). Estos atributos son los siguientes: 1) alcanzable, 2) desafiante; 3) concreto; 4) creíble; 5) que mejore la eficacia; 6) aprendizaje experiencial; 7) orientado a la retroalimentación; 8) divertido; 9) impulsado por la identidad; 10) nivelado; 11) significativo; 12) 
impulsado por la narración; 13) impulsado por la recompensa; 14) simulador; y 15) social. Estos atributos son clasificados en las dimensiones de compromiso cognitivo, emocional y de comportamiento (ver Figura 9).

Los resultados obtenidos indican que el juego propuesto presenta la mayoría de los atributos que potencian el compromiso ambiental en juegos serios (Tabla 1). Además, presenta características, tales como, retos alcanzables, creíbles, retantes y orientados a la retroalimentación que caen en las tres dimensiones, lo que significa que nuestro juego presenta los elementos centrales que potencian la creación simultánea en los usuarios de un compromiso cognitivo, emocional y de comportamiento.

TABLA 1

Lista de control de los atributos del juego S.O.S: Civilizaciones

\begin{tabular}{|l|c|}
\hline Atributos & Valoración \\
\hline Retos alcanzables & $\sqrt{ }$ \\
\hline Desafiante & $\sqrt{ }$ \\
\hline Concreto & $\sqrt{ }$ \\
\hline Creíble & $\sqrt{ }$ \\
\hline Eficaz & $\sqrt{ }$ \\
\hline Aprendizaje experiencial & $\sqrt{ }$ \\
\hline Orientado a la retroalimentación & $\sqrt{ }$ \\
\hline Divertido & $\sqrt{ }$ \\
\hline Impulsado por la identidad & $\mathrm{X}$ \\
\hline Nivelado & $\sqrt{ }$ \\
\hline Significativo & $\sqrt{ }$ \\
\hline Impulsado por la narración & $\sqrt{ }$ \\
\hline Impulsado por la recompensa & $\mathrm{X}$ \\
\hline Simulador & $\sqrt{ }$ \\
\hline Social & \\
\hline
\end{tabular}

Tomado de Ouariachi et al. 2018 


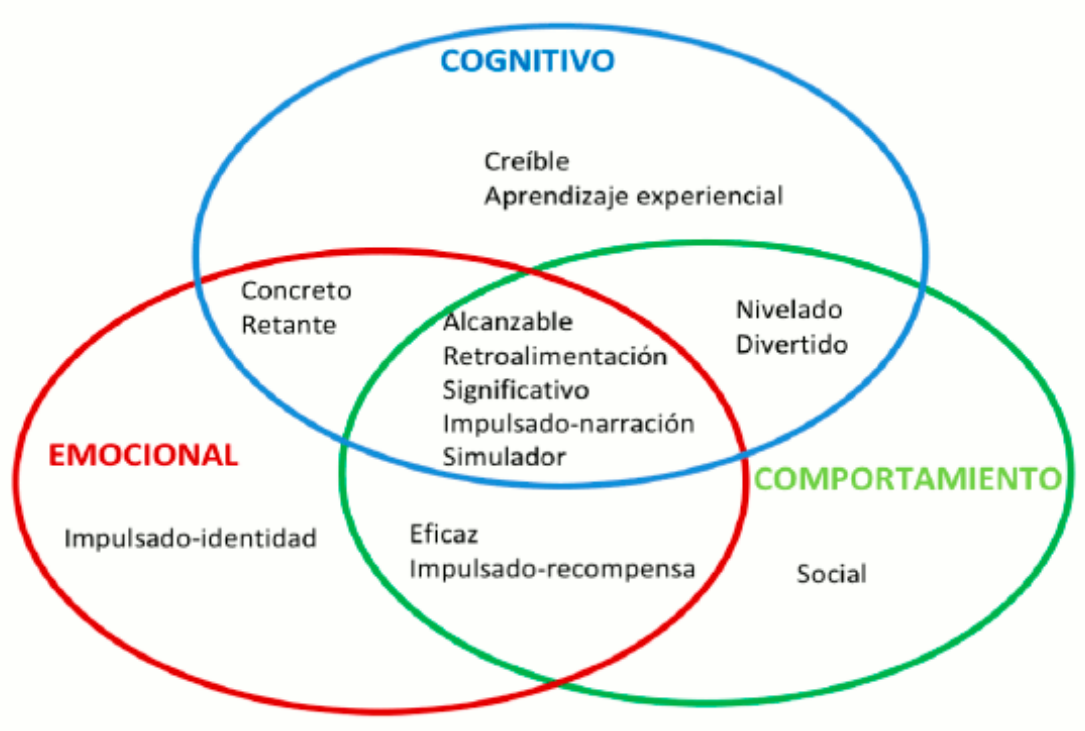

FIGURA 9

Marco de compromiso con el Cambio Climático a través de los juegos serios.

Ouariachi et al., 2018

\section{DisCUSión Y CONCLUSIONES}

Este recurso educativo ha sido desarrollado para contribuir a la formación y conocimiento del alumnado de centros de bachillerato en las problemáticas medioambientales y sociales actuales, a través de los Objetivos de Desarrollo Sostenible y la puesta en valor de los mismos. Cabe mencionar que, a pesar de estar basado en los ODS de la ONU, ha sido difícil encontrar un espacio en el currículum español de secundaria y bachillerato para la formación de los mismos, encontrándose actualmente como una formación difusa (Dieste, Coma y Blasco Serrano 2019). Esta situación es algo paradójica, ya que hablamos de retos sociales y medioambientales de carácter internacional, que por tanto deberían estar considerados dentro del currículum de las distintas etapas educativas. Para cumplir estos objetivos, debemos primero educar en los mismos, para ponerlos así en valor en la sociedad actual y promover la sostenibilidad.

La evaluación del juego S.O.S Civilizaciones en los marcos teóricos examinados indica que este juego tiene un alto potencial para educar y fomentar el comportamiento pro-ambiental. Los resultados obtenidos demuestran que el juego diseñado presenta un equilibrio entre las unidades de motivación extrínseca e intrínseca. Sin embargo, los factores de significado épico y la llamada y la influencia social y sentimiento de pertenencia, pertenecientes al grupo de sombrero blanco, y sólo un factor de sombrero negro, como es el sentimiento de pérdida están valorados muy positivamente, lo cual demuestra que el juego diseñado potencia especialmente los sentimientos positivos. Este hecho podría suponer una debilidad en el diseño del juego, ya que los sentimientos negativos, que, si se diseñan de forma adecuada y consciente, pueden fomentar una modificación urgente del comportamiento y equilibrar las emociones de los usuarios. En cuanto al marco de compromiso al cambio climático y temas ambientales relacionados, el juego diseñado presenta la mayoría de los atributos propuestos por Ouariachi et al. (2018) que potencian el compromiso pro-ambiental en los usuarios. Además, presenta características tales como: retos alcanzables, creíbles, retantes y orientados a la retroalimentación que caen en las tres dimensiones de compromiso, lo que significa que nuestro juego presenta un alto potencial para crear simultáneamente en los usuarios un compromiso cognitivo, emocional y de comportamiento. Además, cuantos más atributos se incluyan en el diseño de la gamificación, más 
fuertes son las conexiones físicas y mentales de los participantes, y mayor es el potencial para influir en el comportamiento humano (Ouariachi et al., 2018).

Teniendo en cuenta las limitaciones de este trabajo, nosotras reconocemos la posible subjetividad de los datos y las implicaciones interpretativas de la metodología utilizada, a pesar del esfuerzo realizado por lograr una alta fiabilidad y validez en el análisis. Además, existe una falta de estudios previos sobre este campo tan específico que dificultan poder contrastar los resultados obtenidos con otros estudios concretos de gamificación. Por otro lado, se anima a los académicos a llevar a cabo un estudio empírico para evaluar si el recurso diseñado en este trabajo tiene realmente potencial de influir en el comportamiento pro-ambiental humano, para ello se recomienda realizar una investigación cualitativa con estudiantes de Bachillerato a través de preguntas abiertas sobre la experiencia del juego (ej. Garrido-Rosales et al., 2020; Vázquez-Vílchez et al., 2021).

\section{MATERiales SUPLEMENTARios}

Anexo1 (pdf)

\section{Agradecimientos}

Agradecemos a la Universidad de Granada la financiación del proyecto de innovación docente PID19-67. También expresamos nuestro agradecimiento por el apoyo financiero al grupo de investigación HUM-613 de la Junta de Andalucía.

\section{ReFERENCIAS}

Alsawaier R.S. (2018) The effect of gamification on motivation and engagement. International Journal of Information Learning Technology 35, 56-79.

Baaden M., Delalande O., Ferey N., Pasquali S., Waldispühl J., Taly A. (2018) Ten simple rules to create a serious game, illustrated with examples from structural biology. PLoS Computational Biology 14(3), e1005955. doi: ht tps://doi.org/10.1371/journal.

Behl A., Sheorey P., Pal A., Veetil A.K.V., Singh S.R. (2020) Gamification in E- Commerce. Journal of Organizational Computing and Electronic Commerce 18, 1-16.

Chou Y.K. (2015) Actionable Gamification: Beyond Points, Badges, and Leaderboards. Createspace Independent Publishing Platform: Milpitas, CA, USA.

Chou J.Y., Lee, E.H. (2014) Reducing Confusion about Grounded Theory and Qualitative Content Analysis: Similarities and Differences. The Qualitative Report 19, 64.

Corner A., Roberts O., Chiari S., Völler S., Mayrhuber E.S., Mandl S., Monson K. (2015) How do young people engage with climate change? The role of knowledge, values, message framing, and trusted communicators. Wiley Interdisciplinary Reviews: Climate Change 6(5), 523-534.

Deterding S., Khaled R., Nacke L., Dixon D. (2011) Gamification: Toward a Definition Chi 2011 Gamification Workshop;12, 1-6.

Dias J. (2107) Teaching Operations Research to Undergraduate Management Students: The Role of Gamification. International Journal of Manaement Education 15, 98-111.

Dieste B., Coma T., Blasco Serrano A.C. (2019) Inclusión de los Objetivos de Desarrollo Sostenible en el Currículum de Educación Primaria y Secundaria en Escuelas Rurales de Zaragoza. Revista Internacional De Educación Para La Justicia Social 8(1). doi: https://doi.org/10.15366/riejs2019.8.1.006. 
Ana González-Robles, et al. Propuesta educativa para promover compromisos ambientales a través de ...

Garrido-Rosales D., Pérez-Fernández B., Vázquez-Vílchez M., Fernández-Oliveras A. (2020) Evaluación de un prototipo de juego de mesa colaborativo para la formación del profesorado en el cambio global. 29 Encuentros de Didáctica de las Ciencias Experimentales y $5^{a}$ Escuelas de Doctorado, 1481-1487.

Gómez Trigueros I.M. (2018) Gamificación y tecnologías como recursos y estrategias innovadores para la enseñanza y aprendizaje de la historia. Educação \& Formação 3(8), 3-16.

Larson K. (2020) Serious Games and Gamification in the Corporate Training Environment: A Literature Review. TechTrend 64, 319-328.

Monroe M.C., Oxarart A., Plate R.R. (2013) A role for environmental education in climate change for secondary science educators. Applied Environmental Education \& Communication 12(1), 4-18.

Ouariachi T., Olvera Lobo M. D., Gutiérrez Pérez J. (2018) A framework for climate change engagement through video games. Environmental Education Research 25(5), 701-716.

Ouariachi T., Olvera Lobo M.D., Gutiérrez Pérez J. (2017) Evaluación de juegos online para la enseñanza y aprendizaje del cambio climático. Enseñanza de las ciencias: revista de investigación y experiencias didácticas 35(1), 193-214.

Sailer M., HomnerL. (2020) The Gamification of Learning: A Meta-Analysis. Educational Psychology Review 32, 77 112.

Shaffer D. W., Halverson R., Squire K. R., Gee J. P. (2005) Video games and the future of learning. WCER Working, 2005-4

Sardi L., Idri A., Fernández-Alemán J.L. (2017) A Systematic Review of Gamification in E-Health. Journal of Biomedical Informatics 71, 31-48.

Schmidt M., Radchuk O., Meinhart, C. (2014) A serious game for public engagement in synthetic biology. En S. Göbel y J. Wiemeyer (Eds) International Conference on Serious Games: Lecture Notes in Computer Science (pp. 77-85), Cham: SpringerWouters P., van Nimwegen C., van Oostendorp,H., y van der Spek E. D. (2013) A metaanalysis of the cognitive and motivational effects of serious game. Journal of Educational Psychology 105 (2), 249-265. https://doi.org/10.1037/a0031311.

Vázquez-Vílchez M., Garrido-Rosales D., Pérez-Fernández B., Fernández-Oliveras A. (2021) Compromiso proambiental del profesorado en formación mediante aprendizaje basado en juegos. En F. Cañada y P. Reis (Eds). Actas Electrónicas del XI Congreso Internacional en Investigación en Didáctica de las Ciencias 2021. Aportaciones de la Educación Cientifica para un mundo sostenible, 1183-1186.

Wu J.S., Lee J.J. (2015) Climate change games as tools for education and engagement. Nature Climate Change 5(5), 413-418.

\section{INFORMACIÓN ADICIONAL}

Para citar este artículo: González-Robles A. y Vázquez-Vílchez M. (2022) Propuesta educativa para promover compromisos ambientales a través de los Objetivos de Desarrollo Sostenible en Bachillerato: el juego S.O.S. Civilizaciones. Revista Eureka sobre Enseñanza y Divulgación de las Ciencias 19(1), 1103. doi: 10.25267/ Rev_Eureka_ensen_divulg_cienc.2022.v19.11.1103 